COMPTES RENDUS

\title{
L'OCCIDENT MÉDIÉVAL (VIII'-XVe SIÈCLE) HISTOIRE ET ANTHROPOLOGIE
}

Christiane Klapisch-Zuber, L'Ombre des ancêtres. Essai sur l'imaginaire médiéval de la parenté. Paris, Fayard, 2000. $16 \times 24,450$ p., ill. (L'Esprit de la cité).

La généalogie fait peur aux historiens : elle rappelle trop l'utilisation partisane et orientée de l'histoire, destinée à agencer des lignées ou à légitimer des successions. Face à la vogue des arbres généalogiques de toute espèce, fruits d'associations d'amateurs éclairés ou des puissantes bases de données informatiques élaborées par les Mormons, l'historien universitaire ne peut alors réagir qu'avec une certaine gêne, tant il sait ce que cette perpétuelle quête des « racines » a d'artificiel, voire d'idéologique.

Le livre de Christiane Klapisch-Zuber marque le retour de la généalogie dans la réflexion historique, non plus comme fin, mais comme objet. Plus précisément, c'est moins le discours généalogique lui-même qui l'intéresse que la forme figurée qu'il prend, celle de l'arbre, qui s'est peu à peu imposée dans notre imaginaire. Ce travail donne donc un éclairage très original sur l'histoire de la famille et de la parenté médiévales, un courant de recherche dont Klapisch-Zuber est, depuis presque trois décennies, l'une des plus illustres représentantes. Ce livre est aussi la concrétisation de toute une série de réflexions menée depuis une dizaine d'années, notamment à l'École des hautes études en sciences sociales, sur l'image médiévale et son utilisation par l'historien. En faisant le choix de l'image comme source principale, Klapisch-Zuber dépasse le stade de la simple réflexion méthodologique en montrant très concrètement ce que l'étude attentive de l'image peut apporter de neuf au travail de l'historien. Elle remet de la sorte en lumière des images souvent négligées par les historiens des textes qui n'en voyaient que le contenu, et par les historiens de l'art pour qui ces dessins souvent embrouillés ne présentaient que peu d'intérêt.

Pour un médiéviste, l'iconographie de l'arbre généalogique évoque inévitablement le thème de l'arbre de Jessé qui envahit vitraux et manuscrits depuis le XII ${ }^{\mathrm{e}}$ siècle. De là à en faire l' ancêtre des arbres généalogiques de la Renaissance, il n'y a qu'un pas que l'on franchissait jusqu'alors allègrement. Klapisch-Zuber s'attache à montrer la fragilité de ce raccourci. D'abord parce que l'assimilation de la prophétie d'Isaïe à la généalogie du Christ est loin d'être systématique et n'intervient qu'assez tardivement, au prix d'ailleurs d'une exégèse délicate destinée à harmoniser la 
double généalogie de Matthieu et de Luc. Ensuite parce que l'iconographie de Jessé n'est qu'une des nombreuses tentatives pour mettre en image une lignée.

L'étude de Klapisch-Zuber s'inscrit donc dans un large Moyen Âge, même si la floraison d'arbres généalogiques du début de la Renaissance reste le point de départ et l'aboutissement de son travail. Quatre grandes parties conduisent ainsi le lecteur depuis la mise en scène des portraits des défunts dans les atria des grandes familles romaines jusqu'aux complexes généalogies imprimées de l'époque moderne. L'auteur s'attache tout d'abord à montrer la lente mise en place, entre le $\mathrm{IX}^{\mathrm{e}}$ et le $\mathrm{XII}^{\mathrm{e}}$ siècle, d'une véritable « langue graphique » de la parenté et de la lignée, autour des arbres de consanguinité élaborés par les juristes, ou encore des spectaculaires généalogies bibliques illustrant le Commentaire de l'Apocalypse de Beatus de Liébana dans l'Espagne du $\mathrm{X}^{\mathrm{e}}$ siècle. Dans le même temps, l'espace germanique voit naître les premières représentations généalogiques sous forme d'arbres, notamment pour la dynastie des Welfes, à la fin du XII ${ }^{\mathrm{e}}$ siècle. Mais l'élan décisif est alors donné, selon l'auteur, par la théologie parisienne, et surtout par le Compendium historiae in genealogia Christi de Pierre de Poitiers, successeur de Pierre le Mangeur à l'école de la cathédrale de Paris. Cet abrégé est d'ailleurs annexé dans la plupart des manuscrits à la célèbre Histoire scolastique de Pierre le Mangeur, ce qui lui assure une exceptionnelle diffusion. Il s'agit avant tout d'un outil didactique qui déploie la généalogie du Christ sur un rouleau de parchemin, combinant notices et médaillons autour d'un axe central. Dans la seconde partie de son livre, Klapisch-Zuber insiste sur l'influence qu'a pu avoir ce schéma sur la diffusion des représentations généalogiques entre le $\mathrm{XII}^{\mathrm{e}}$ et le $\mathrm{XIV}^{\mathrm{e}}$ siècle. Le modèle se retrouve assez nettement dans les schémas qui accompagnent les Chroniques universelles et qui cherchent à mettre en parallèle l'histoire laïque et l'histoire spirituelle dans une vision globale du temps. L'image devient alors le moyen d'organiser non seulement une lignée mais l'ensemble de l'histoire humaine. Elle devient ainsi très vite instrument de propagande au service des grands débats dynastiques. On voit alors apparaître au XIII ${ }^{\mathrm{e}}$ siècle de très nombreuses images destinées à légitimer en France la dynastie des Capétiens, ou en Angleterre celle des Plantagenêts. Il s'agit moins de montrer une filiation que de justifier la continuité dynastique. Le modèle sera aussi utilisé à la fin du Moyen Âge dans la construction des histoires nationales, à la recherche d'ancêtres mythiques ou bibliques.

La forme de l'arbre s'impose alors pour représenter la généalogie. Dans le contexte des débats nominalistes sur les rapports entre la réalité et sa désignation, la chose ne va pas forcément de soi. Klapisch-Zuber consacre la troisième partie de son livre aux problèmes et aux débats suscités par l'adoption de l'arbre dans la représentation généalogique. Elle rappelle tout d'abord l'invasion de la métaphore de l'arbre dans tous les domaines de la pensée : l'arbre des âges de la vie, « l'arbre des batailles », l'arbre de vie, l'arbre de la croix, etc. L'image évoque tout à la fois la germination de la vie, l'étagement du temps, la cohésion d'une communauté monastique, ou encore un moyen de classification. On connaît par exemple le succès du thème chez les Franciscains, comme en témoignent le Lignum vitae de saint Bonaventure ou l'Arbor vitae d'Ubertin de Casale. Même Joachim de Flore utilise la métaphore de l'arbre pour illustrer sa vision dynamique de l'histoire. 
L'adoption du thème pour la représentation de la généalogie n'est donc qu'un élément de cette diffusion de l'arbre dans la pensée. Pourtant, l'image pose un certain nombre de problèmes, aux juristes et aux artistes, lorsqu'il s'agit de représenter un arbre de parenté dont les racines - Ego - se trouvent au sommet. Homo arbor conversa - «l'homme est un arbre inversé »- trouve-t-on chez Platon, une métaphore qui justifie alors le renversement de l'arbre et suscite des tentatives artistiques originales et parfois embrouillées, comme la représentation de l'ancêtre au sommet d'un tronc dont les branches pendantes portent les descendants. Quoi qu'il en soit, aux $\mathrm{XV}^{\mathrm{e}}$ et $\mathrm{XVI}^{\mathrm{e}}$ siècles, l'arbre généalogique est devenu un élément fondamental de la mise en scène du pouvoir, comme en témoignent alors les entrées royales, en France ou en Angleterre.

La quatrième partie évoque enfin ce triomphe de l'arbre généalogique au début de la Renaissance, grâce à l'imprimerie tout d'abord, mais aussi par l'utilisation spectaculaire qu'en font les Habsbourg et notamment Maximilien I ${ }^{\mathrm{er}}$, aboutissement d'une stratégie politique fondée sur les alliances familiales. Les généalogies modernes, tout en gardant l'idée de l'arbre, évolueront vers une schématisation croissante, conduisant aux symboles graphiques des ethnologues du Xx $\mathrm{x}^{\mathrm{e}}$ siècle.

Le livre de Klapisch-Zuber va donc bien au delà d'une simple étude sur l'iconographie des arbres généalogiques. C'est toute la pensée médiévale qui se trouve mobilisée autour de l'arbre, au service du pouvoir, d'une idée ou d'une communauté. Fruit de quinze années de travail, L'Ombre des ancêtres est un livre extrêmement savant qui progresse pas à pas parmi des dizaines d'images, dont la plupart n'ont pas pu trouver leur place dans les cinquante illustrations que compte l'ouvrage. Le lecteur est donc contraint de stimuler son propre imaginaire pour se représenter la plupart des exemples évoqués par l'auteur - un inconvénient matériel qui handicape toute étude historique mobilisant un important corpus iconographique. À cette contrainte éditoriale s'ajoute le légitime souci de l'auteur de rendre toute la complexité de la question, de s'écarter des évidences qui s'imposent trop facilement en matière de généalogie. Non, l'arbre n'est pas la seule façon de représenter la généalogie, et elle n'est pas non plus la plus évidente. Non, l'arbre ne dit pas nécessairement la filiation, mais parfois aussi l'unité spirituelle d'une communauté, à l'exemple des arbres franciscains ou dominicains. Klapisch-Zuber entraîne donc son lecteur dans une forêt que cachait jusqu'alors le célèbre arbre de Jessé, et dans lequel le risque est grand de se perdre. Sachant aborder l'image en tenant compte de sa spécificité, elle est attentive à montrer les enjeux formels de son évolution : le passage du rouleau au codex qui brise la continuité des frises, l'apparition de l'imprimerie qui oblige à une simplification des lignes, le choix des formes graphiques, etc. On peut seulement regretter la brièveté des bilans et des conclusions, ou la rareté des éclairages plus généralistes qui permettraient au lecteur de trouver plus facilement ses repères dans cette profusion d'arbres.

Il reste que cet ouvrage apparaît fondamental pour aborder la complexité de «l'imaginaire médiéval de la parenté », et pour comprendre le poids d'une représentation toujours présente avec succès dans l'imaginaire contemporain. Fruit des tentatives médiévales pour mettre en ordre l'histoire humaine, l'image de l'arbre s'est imposée au point de faire oublier ce qu'elle avait de problématique et d'idéologique. 
L'Ombre des ancêtres montre la complexité des relations entre un discours et sa mise en image, lorsque la forme agit à son tour sur les façons de penser et de dire le réel.

Paul PAYAN

Éric Palazzo, Liturgie et société au Moyen Âge. Paris, Aubier, 2000. 13,5×22, 279 p., index, ill. (Collection historique).

Ce livre d'Éric Palazzo, professeur d'histoire de l'art du Moyen Âge à l'université de Poitiers, ne prétend pas être une somme, mais une approche synthétique des problèmes posés à l'historien par les multiples rôles de la liturgie au Moyen Âge. Il se recommande d'une interdisciplinarité visant à montrer comment l'étude de la liturgie, hors du cercle des théologiens ou d'historiens des religions qui l'a longtemps accaparée, se nourrit des renouvellements issus de l'utilisation des outils fournis à l'histoire et à l'histoire de l'art par l'anthropologie et la sociologie. Les rappels et les citations d'ouvrages théoriques qui parsèment l'ouvrage résument d'ailleurs presque à eux seuls un siècle d'importations de concepts anthropologiques vers l'histoire, depuis l'ensemble des sciences humaines par les historiens, de Claude Lévi-Strauss et des rites dogons chers à Marcel Griaule jusqu'à Pierre Bourdieu, en passant par Norbert Elias ou Gaston Bachelard, sans oublier Erwin Panofsky et Ernst Kantorowicz, saints patrons de l'histoire de l'art et des idées politiques du Moyen Âge occidental.

On aura d'ores et déjà compris que dans les dimensions relativement restreintes de l'ouvrage, l'auteur vise à évoquer le plus de champs d'applications possibles de cette foisonnante interdisciplinarité. Aussi celui-ci est-il divisé en neuf sections autonomes, que l'on peut regrouper en trois ensembles de cohésion et de longueur inégales. Comme il le rappelle en introduction (p. 11-16), l'auteur s'appuie tantôt sur ses propres recherches, tantôt résume des analyses de différents chercheurs.

Une première partie, articulée en quatre chapitres, répond aux promesses du titre général de l'ouvrage, en traitant spécifiquement de la liturgie dans la société. «La messe dans la société médiévale » (p. 17-39) insiste particulièrement sur la liturgie de l'eucharistie comme facteur de cohésion sociale, en reprenant au passage de récentes études s'attachant à mettre en évidence le rôle social du banquet funéraire dans l'antiquité. «La liturgie des sacrements » (p. 40-57) s'essaie à examiner les évolutions du baptême et de la confirmation, du mariage, de l'ordination. Enfin, «Les rites autres que les sacrements » (p. 58-77) contient des développements sur les processions et la dédicace, et «La liturgie des fidèles » (p. 78-97) tente de réévaluer le délicat équilibre entre les acteurs passifs et actifs, religieux et laïques, hommes et femmes, des différentes liturgies.

La seconde partie, comprenant deux chapitres, voudrait recréer un espace-temps de la liturgie (chap. V : « La liturgie et le temps », p. 98-123; chap. VI : « La liturgie et l'espace », p. 124-149). Le temps liturgique de l'année ou de la semaine est mis 
en relation avec d'autres temps de la réalité ou de l'imaginaire médiévaux, avec un hommage appuyé aux analyses de Jacques Le Goff ; le développement sur la liturgie et l'espace, est, pour sa part, centré sur le problème des liturgies de l'extérieur, plus particulièrement sur la fonction de l'autel portatif - source de réflexions et de tensions peu connues au Moyen Âge central, et thème de prédilection de l'auteur qui annonce un livre sur cette question.

La transition s'opère naturellement avec les trois derniers chapitres, qui forment autant de sections relativement autonomes, renvoyant néanmoins toutes aux « miroirs » iconographiques, hagiographiques ou politiques de la liturgie comme image et modèle idéal de la société. « La liturgie et les images » (chap. VII, p. 150-176) est l'occasion d'une mise au point méthodologique qui se veut une condamnation des lectures fonctionnalistes de l'iconographie liée aux liturgies. Elle est suivie d'une série de démonstrations, mettant en relief le rôle explicatif de différentes sources iconographiques dans leur encadrement liturgique originel. « La liturgie et les saints » (chap. VIII, p. 177-193) rappelle le riche contenu liturgique de la littérature hagiographique et les modèles de perfection liturgique qu'elle propose à ses destinataires. Enfin, « La liturgie et le politique » (p. 194-212) évoque l'intrication entre les rituels du pouvoir et ceux de l'Église, notamment par le biais des rituels des couronnements royaux. L'ouvrage se termine, en conclusion, par un vibrant appel à la poursuite de l'interdisciplinarité et de la sortie des études liturgiques hors d'un ghetto purement religieux.

En abordant cet ensemble de questions très diverses, le livre de Palazzo réussit son objectif de présenter au moins partiellement les différentes voies explorées par les chercheurs en relation avec les problèmes posés par la liturgie médiévale. Son utilisation abondante des recherches récentes menées par Irène Rosier dans le domaine de la linguistique médiévale (analyses linguistiques des grammairiens des $\mathrm{XII}^{\mathrm{e}}$ et XIII ${ }^{\mathrm{e}}$ siècles sur les formules sacramentelles, p. 31-35), ou des fines analyses de Jacques Chiffoleau sur les processions comme cadres de la religion flamboyante de l'automne du Moyen Âge (p. 68-71), pour ne pas parler de l'évocation des travaux de Jacques Le Goff sur les différents temps (temps du purgatoire, temps des marchands) de la société médiévale, rappelle utilement les chantiers défrichés au cours de ces cinquante dernières années.

On peut en revanche se demander si la volonté proclamée par l'auteur de contextualiser l'étude de la liturgie médiévale, d'en faire un objet d'histoire à part entière, au-delà des études linguistiques ou théologiques, n'entre pas, à partir d'un certain point, en contradiction avec son utilisation massive de concepts anthropologiques qui tendent au contraire à lui enlever un contexte historique précis, en incluant des comparaisons qui vont de l'antiquité ou de l'ethnologie africaine aux temps contemporains. Cette question se pose avec d'autant plus d'acuité que l'auteur a adopté un cadre chronologique très large, allant $d u v^{e}$ au $X^{e}$ siècle, et qu'il utilise sans cesse des documents littéraires, normatifs ou iconographiques d'époques très diverses.

Ce n'est pas que l'auteur ne souligne pas parfois les évolutions, par exemple en ce qui concerne le problème du symbolisme et de la pensée de l'eucharistie au Moyen Âge central, mais ses analyses, centrées sur un «temps long de la liturgie », 
donnent parfois l'impression de négliger d'autres temps - plus courts, même au Moyen Âge - tels que celui de la réflexion philosophique ou linguistique. Ainsi, pages 33-34, après un rappel des spéculations linguistiques de Bérenger de Tours (1000-1088), il passe sur deux bons siècles de réflexion philosophique et linguistique, pour arriver aux théories d'un Jean Duns Scot (vers 1265-1308), « peu de temps après » le premier. La volonté de l'auteur de résumer le maximum d'analyses différentes est sans doute à l'origine de tels raccourcis. Elle se reflète parfois dans des formulations ramassées qu'un lecteur non averti aura peut-être du mal à décoder. Ainsi, dans le même développement, on peut lire que Duns Scot «intervint de façon décisive dans ce débat [celui de l'énoncé du discours sacramentel]. Pour cela, il allait utiliser les problèmes logiques et sémantiques de la formule de consécration eucharistique afin d'en tirer des conclusions sur l'histoire de la grammaire et de la logique médiévales » (p. 34), en une formulation qui confond nettement les récentes avancées de l'histoire de la grammaire et de la logique médiévale avec leurs objets d'étude respectifs.

Plus importante pour l'étude de la liturgie proprement dite, est la question de la contextualisation chronologique. Si l'antiquité tardive est une période essentielle pour l'étude des fondements et de la mise en place de la liturgie, on peut se demander dans quelle mesure la spécificité désormais bien reconnue de cette période permet à l'historien d'inclure, dans un ouvrage par ailleurs très synthétique, des analyses sur le rituel et les lieux du rituel de l'Occident romain et romano-byzantin des ve, $\mathrm{VI}^{\mathrm{e}}$ et $\mathrm{VII}^{\mathrm{e}}$ siècles, mais aussi une réflexion sur les trois derniers siècles du Moyen Âge. L'importance des développements sur le rôle de la liturgie à l'époque carolingienne, si fondamentale au regard des réformes ou des unifications lancées par les cadres politiques et religieux de l'empire à cette époque, amène d'ailleurs à poser également le problème pour les siècles qui s'étendent entre ces deux périodes.

Là encore, la volonté de traiter le maximum de thèmes dans un espace réduit a sans doute conduit l'auteur à renoncer à une mise en perspective problématique qui aurait considérablement allongé son propos. Mais en rappelant au début, au milieu (notamment pour la question de la place des femmes dans la liturgie, p. 95-97) et à la fin de son ouvrage les enjeux d'une réflexion sur la liturgie pour l'époque contemporaine, l'auteur ne montre-t-il pas les limites de l'historicisation d'un tel sujet dans une approche purement chrétienne ? Pourtant, les nombreuses analyses qui traitent de l'enjeu des unifications ou de contrôles de la liturgie par l'Église dans sa lutte pour le contrôle de la société à différentes époques suggèrent admirablement la richesse potentielle d'une histoire en continu des inflexions de la liturgie et de ses changements dans la société, entre réflexion et pratique, sur la très longue durée.

Entre les mises en place et les « inculturations » de l'antiquité tardive, les unifications et les fastes monastiques de l'ère carolingienne, les controverses intellectuelles des $\mathrm{XI}^{\mathrm{e}}, \mathrm{XII}^{\mathrm{e}}$ et $\mathrm{XIII}^{\mathrm{e}}$ siècles et les liturgies citadines de la religion flamboyante, n'y a-t-il pas place pour différentes histoires de la liturgie ? C'est ce que la diversité des champs évoqués par Palazzo peut faire légitimement espérer. 
Jean-Claude Schmiтt, Le Corps, les rites, les rêves, le temps. Essais d'anthropologie médiévale. Paris, Gallimard, 2001. 14 ×23, 449 p., 21 ill. (Bibliothèque des histoires).

Le titre composite du dernier ouvrage de Jean-Claude Schmitt l'indique comme une évidence : il ne s'agit pas, comme dans les précédentes livraisons de ce grand médiéviste, de proposer la synthèse d'une recherche aboutie. Au contraire : par la réunion dans ce recueil de 17 articles dont la parution s'est étalée sur une trentaine d'années, Schmitt entend faire la lumière sur son parcours intellectuel d'historien du Moyen Âge occidental. Surtout, au-delà de la multiplicité des objets historiques sur lesquels il a été amené à réfléchir depuis les années 1970, l'auteur entend redonner quelques-uns des principaux jalons qui ont marqué l'introduction de l'anthropologie dans la pratique professionnelle des médiévistes (notamment au sein de l'historiographie française). Non qu'il se présente comme l' «inventeur » d'une méthode qui a permis de renouveler en profondeur les problématiques de nombreux champs de l'historiographie médiévale - Schmitt rappelle suffisamment la dette qu'il a contractée à cet égard envers Marc Bloch et Jacques Le Goff, pour ne citer qu'eux -, mais il entend rappeler, d'une part, que cette entrée de l'anthropologie dans le champ historique ne s'est pas faite sans heurts, et, d'autre part, qu'elle est encore loin à ce jour d'avoir livré tous ses fruits.

La préface est l'occasion d'une remise en perspective historiographique éclairante, notamment pour la jeune génération de médiévistes. L'introduction de modes de questionnement anthropologique dans le champ des études historiques a eu pour principal bénéfice d'avoir ponctuellement décloisonné des domaines de recherche auparavant trop exclusifs les uns des autres, alors même que l'émiettement des objets de recherche tendait à se confirmer. En privilégiant l'étude des systèmes de représentations, elle a permis aux médiévistes de se départir, autant que faire se pouvait, de leurs réflexes ethnocentriques et de complexifier leurs approches. Par exemple, alors que l'on pensait généralement jusque-là la religion (terme du reste impropre) comme une sphère autonome du politique et de l'économique, le changement de point de vue a permis de mettre en évidence à quel point tout l'ensemble des représentations et des pratiques sociales se nourrissait au Moyen Âge du christianisme. L'approche anthropologique, en instituant une dimension comparatiste jusque-là quasi inexistante dans les études historiques, a largement fait prendre conscience aux historiens de la relativité de leurs objets d'études, de leurs catégories de pensée et, par la même, de leur vocabulaire.

L'intérêt porté aux mots utilisés est du reste l'un des principaux fils d'Ariane qui innervent le présent recueil et assurent sa cohérence. La critique lexicale est en effet pour l'historien le premier moyen dont il dispose pour se distancier de son sujet. Ce n'est ainsi pas un hasard si l'ouvrage commence par une communication au titre en apparence provocateur - «Une histoire religieuse du Moyen Âge est-elle possible ?» (1994) - dans laquelle l'auteur remet en cause la pertinence d'un certain nombre de notions trop longtemps admises, sans regard critique, par une historiographie chrétienne qui percevait sans doute trop son objet d'étude - la religion chrétienne au Moyen Âge - comme un héritage. Est-il en effet légitime d'user du 
terme de « religion » alors que son précédent latin - religio - n'était en rien doté du sens moderne qui lui est seulement attribué au XVIII ${ }^{\mathrm{e}}$ siècle ? Faut-il en outre toujours évoquer sans précaution les superstitions, alors que le vocable traduit le jugement de valeur de l'institution ecclésiale ? Doit-on enfin toujours faire de la magie la manifestation d'un esprit irreligieux, voire, pis, areligieux ?, etc. De nombreuses recherches, passées et actuelles, montrent que la prise en compte de cette relativité terminologique est l'un des plus sûrs moyens de ne pas verser ipso facto dans l'anachronisme, même s'il reste souvent, dans la pratique, difficile de se départir complètement des champs lexicaux contemporains.

Schmitt procède de fait à de multiples reprises (la plupart du temps dans des articles programmatiques) à des analyses lexicales qui servent de préalables à tous ses développements ultérieurs. Il s'interroge ainsi, dans le second article du recueil ( «La notion de sacré et son application à l'histoire du christianisme médiéval », 1991), sur les ressorts du « sacré » dans la société chrétienne médiévale, en isolant ses spécificités par rapport aux manifestations qui sont les siennes dans les contextes propres au paganisme et au judaïsme antiques. Il montre ainsi le rôle central joué par l'Église dans sa définition et dans la reconnaissance (ou non) de ses manifestations, tout en insistant sur les bornes que la critique rationnelle des clercs médiévaux lui a progressivement posées, dans lesquelles d'aucuns ont vu la germination d'un futur esprit laïque et encore d'un « désenchantement » du monde occidental. Dans une démarche qui se veut toujours comparatiste (le monde antique occidental sert le plus souvent de référent), l'historien discute ensuite, dans une troisième étude («Problèmes du mythe dans l'Occident médiéval », 1988), la notion de « mythe » dans le contexte chrétien antique et médiéval, avant d'en venir, dans une quatrième («La croyance au Moyen Âge », 1995) et une cinquième («Du bon usage du Credo », 1981), à la notion fondamentale de «croyance ». Comment en effet se définit une vraie et une fausse croyance ? Selon quelles modalités l'Église a-t-elle suscité l'adhésion de ses membres ? Quel espace critique enfin existait pour les différentes couches de la société médiévale face à l'orthodoxie imposée par l'élite lettrée?

Des réponses à ces questions sont apportées par le biais d'études micro-historiques. L'angle d'attaque privilégié de l'auteur, dans ce domaine, est l'étude des exempla, ces courtes histoires édifiantes utilisées par les prédicateurs pour renforcer la valeur exemplaire de leurs sermons. Ceux-ci permettent, si on les soumet à une analyse structurelle, d'éclairer les rapports ambivalents qu'entretiennent les différents niveaux de culture au sein de la société médiévale, et de critiquer radicalement la dichotomie trop simpliste qui a longtemps opposé dans l'historiographie « culture savante » et «culture populaire ». L'auteur propose ainsi, avec les sixième («Les traditions folkloriques dans la culture médiévale », 1981), huitième («La parole apprivoisée », 1979) et neuvième («Les masques, le diable, les morts », 1986) articles, différents cas d'espèce, en suivant une méthode dont la célèbre communication " "Jeunes" et danse des chevaux de bois » (qui date de 1976 et correspond au septième article du recueil) présentée à Fanjeaux au terme d'un débat aussi houleux que passionné a valeur de manifeste. Il ressort, dans chacune de ces études ponctuelles, que les relations entre l'élite lettrée et la masse des illitterati, loin 
d'être aussi unilatérales ou aussi conflictuelles que l'on a bien voulu le croire parfois, étaient au contraire l'objet et l'occasion d'intenses échanges et de savants jeux de contaminations. La difficulté, pour en rendre compte, tient au fait que « la parole est apprivoisée » par les clercs, et ce n'est donc que par la confrontation des textes au sein de séries documentaires suffisamment denses que la critique historique parvient à démêler l'écheveau en apparence inextricable des réélaborations textuelles successives.

Les relations entre groupes sociaux aux modes de représentations différents mais perméables les uns aux autres posent en contrepoint la difficile question de l'individuation: quelle place reste-t-il, dans la société d'ordres, pour la sphère individuelle ? Comment, par ailleurs, saisir ce qui fait la part irréductible de chacun ? À quand enfin faut-il faire remonter l'émergence de l'individu, si tant est qu'il faille la situer à une date déterminée ? Schmitt reprend la question dans le dixième article du recueil («La "découverte de l'individu". Une fiction historiographique ? », 1989) qui, après un rappel historiographique, replace le débat dans le champ lexical en discutant la pertinence, pour le Moyen Âge, de la notion d' « individu » et montre que sa découverte ne peut être appréhendée, dans cette société façonnée par le christianisme, que dans la combinaison complexe et mouvante que forment les concepts différenciés d'individu, de personne et de sujet.

L'un des moyens les plus sûrs a priori de toucher la subjectivité des médiévaux et de cerner quels rapports elle entretient avec les différents champs de la vie chrétienne (rapports intimes avec Dieu ou le diable, à la sexualité, à la mort et à l'au-delà, etc.) est l'étude de leurs rêves ; mais celle-ci se heurte, comme le rappelle l'auteur dans le onzième article («Les rêves de Guibert de Nogent », 1983, dont la lecture peut-être complétée par le douzième article, «Le sujet du rêve », 1999), à de nombreux écueils. Toutes les époques historiques, en effet, ne se prêtent pas de la même manière à une telle recherche. Au haut Moyen Âge par exemple, les modèles hagiographiques des songes sont trop prégnants pour qu'une spécificité individuelle puisse être perceptible, et ce n'est véritablement qu'au XII ${ }^{\mathrm{e}}$ siècle, en même temps que la confession auriculaire entre dans les mœurs et que le genre autobiographique (dans la lignée des Confessions de saint Augustin) retrouve ses lettres de noblesse, que les relations oniriques s'individualisent et s'affranchissent, en partie, des stéréotypes antérieurs. L'étude des rêves de Guibert de Nogent est d'une part l'occasion d'une mise au point sémantique qui recoupe les différents champs du rêve (distinction entre visio, somnium et phantasma), et, d'autre part, d'une étude structurelle des expériences oniriques (ici à l'échelle d'une vie) dans laquelle peuvent être intégrés les apports de la psychanalyse. Cette dernière permet certes de dévoiler une part de ce qui fait le caractère particulier de Guibert ; mais, aussi loin que l'on souhaite descendre dans les innombrables strates de sa conscience et de son inconscient, il ne peut y avoir en dernier lieu de découverte d'un moi chrétien que dans le jeu des relations qui unit la créature à son Créateur : l'introspection, à l'époque médiévale, ne saurait trouver de justification en elle-même.

Le rêve, lieu privilégié de la relation personnelle avec la divinité, permet par exemple au chrétien qui sait l'interpréter de connaître par avance l'heure de sa mort ou d'être fixé sur son destin post mortem. La relation onirique pose ainsi la 
question connexe de la façon dont était perçu le rapport au corps et au temps dans la société médiévale. C'est pourquoi l'auteur regroupe, au terme de son recueil, deux séries d'articles qui explorent certains aspects de ces deux grands champs d'étude : la première, constituée des treizième ( «Corps malade, corps possédé », 1986) et quatorzième articles ( «Le corps en chrétienté », 1998), analyse les résonances symboliques que pose la question de la corporéité en contexte chrétien ; la seconde, qui comprend les quinzième ( «Temps, folklore et politique au XII ${ }^{\mathrm{e}}$ siècle », 1984), seizième («De l'attente à l'errance. Genèse médiévale de la légende du Juif errant », 2000) et dix-septième ( «'appropriation du futur », 2000) articles, s'attache à la perception que les médiévaux pouvaient avoir du temps, et notamment de l'avenir, objet de toutes les attentes, aussi bien matérielles qu'eschatologiques.

$\mathrm{Au}$ terme de ce tour d'horizon rapide, la mutiplicité et la cohérence des objets d'étude du présent recueil a de quoi impressionner. Toutefois, il ne faut pas s'y tromper : Schmitt n'a pas pour ambition de faire le bilan d'une carrière qui est loin d'être close ; au contraire, en réaffirmant avec force la richesse d'une approche interdisciplinaire, son ouvrage est une invitation destinée aux médiévistes actuels pour qu'ils explorent des voies tout juste entrouvertes.

Julien VÉRONÈSE

Philippe SÉNAC, L'Occident médiéval face à l'Islam. L'image de l'autre. 2e éd. revue, Paris, Flammarion, 2000. $15 \times 21,198$ p., bibliogr., index, cartes, ill. (Histoire de).

La réédition de l'ouvrage de Philippe Sénac, paru en 1983 sous le titre L'Image de l'autre (Paris, Flammarion), souligne le goût toujours prononcé du grand public pour une histoire des mentalités écrite en miroir, et l'intérêt accordé à l'Islam médiéval pour ses apports à la construction historique de l'Occident chrétien. Livré sous un titre plus explicite, le texte original est enrichi d'une bibliographie rajeunie.

Le projet de l'auteur est ambitieux. C'est un vaste chapitre de l'histoire des mentalités médiévales qui est abordé ici, convoquant la trame des événements militaires, les traces de la culture matérielle, comme l'histoire doctrinale et intellectuelle de l'Occident chrétien, maniant les sources iconographiques aussi bien que les textes classiques, mobilisant, enfin, les grandes figures de ce drame, de Charlemagne à Frédéric II, de Pierre le Vénérable à Raymond Lulle...

Comme le reconnaît bien volontiers Sénac dans la préface de cette seconde édition, c'est cependant le royaume de France qui est ici le mieux servi, tant pour l'iconographie, matériau peu abondant et fort méridional (on regrettera d'ailleurs l'absence de référence, dans le texte, aux quelques planches non numérotées qui illustrent cette image de l'autre) que pour la littérature, où la seule Chanson de 
Roland se taille une part de choix. L'auteur n'en conduit pas moins son propos de l'Andalousie à la Sicile, et jusqu'à Constantinople, esquivant cependant en grande partie l'histoire méditerranéenne des villes italiennes.

Sénac décrit l'émergence, l'hégémonie et le recul enfin, d'une image fantasmatique de l'Islam, tronquée et infidèle, délibérément entretenue et diffusée par l'Église romaine, qui s'y arc-boute d'autant plus qu'elle est progressivement démentie par la réalité des échanges culturels et matériels. L'histoire de cette image épouse la chronologie classique de l'Occident médiéval. À la crainte de l'Islam guerrier, que fait résonner au haut Moyen Âge la faveur accordée aux commentaires de l'Apocalypse, succède bientôt la volonté de lutter contre l'hérésie, sous toutes ses formes, par la réfutation et la croisade. La crise de la fin du Moyen Âge, malgré le renouveau du «péril turc », éloigne au contraire de l'Occident, déjà chassé d'Orient, cette obsession de l'autre qui semble avoir tant guidé les attitudes antérieures. Histoire optimiste en définitive, qui voit la méconnaissance délibérée de l'adversaire religieux reculer devant les progrès des contacts profanes et de l'esprit critique.

On suivra volontiers l'auteur pour le primat accordé à la parole de l'Église dans l'élaboration de cette image de l'autre, tout en s'étonnant de la discrétion avec laquelle est abordé le rôle de cette altérité dans la construction doctrinale de la chrétienté occidentale. L'ouvrage de Dominique Iogna-Prat, Ordonner et exclure. Cluny et la société chrétienne face à l'hérésie, au judaïsme et à l'islam, 1000-1150 (Paris, Aubier, 1998) manque ainsi à l'appel.

Sénac souligne, par ailleurs, combien la vision caricaturale d'un Islam diabolisé est d'emblée concurrencée par la fascination matérielle, l'appétit intellectuel (la question de l'averroïsme parisien aurait sans doute mérité d'être traitée à la lumière des travaux d'Alain de Libéra, absents de la bibliographie) et la curiosité suscités par ce qui est entrevu des sociétés islamiques. Il est cependant douteux que l'imagerie orientaliste soit en germe dans la littérature du Moyen Âge central : c'est moins la question du harem qui se pose alors, que celle de l'esclavage, structure portante fondamentale des pouvoirs dans l'Islam médiéval. De même, il n'est pas sûr que les deux visions concurrentes dégagées par l'auteur aient été à ce point antagoniques : qu'on songe simplement à l'importance que conserve l'idée de croisade, longtemps après la chute de Saint-Jean-D'Acre, dans les milieux marchands italiens installés pourtant durablement en Orient.

Au total, l'ouvrage a pour principal mérite d'apporter une intelligibilité globale aux traces éparses laissées par une des altérités fondatrices de l'Occident médiéval. Il rappelle utilement combien l'image de l'Islam en Occident a une histoire longue et contrastée, au gré de circonstances qui ne sont pas, loin s'en faut, toutes orientales.

Julien LOISEAU 
Penser le pouvoir au Moyen Âge, $V I I I^{e}-X V^{e}$ siècle. Études d'histoire et de littérature offertes à Françoise AUTRAND. Textes réunis par Dominique BouTET et Jacques VERGER. Paris, Éditions Rue d'Ulm, 2000. $16 \times 24$, 444 p., ill., bibliogr.

Afin de composer ce beau recueil d'études offertes à Françoise Autrand, Dominique Boutet et Jacques Verger ont réuni autour d'eux 18 auteurs parmi les plus grands noms de la recherche médiévale, français (Colette Beaune, Claude Gauvard, Philippe Contamine, Jean-Philippe Genet...) ou étrangers (Peter S. Lewis, Werner et Anke Paravicini). Autrand ayant consacré ses recherches à l'histoire politique du royaume de France pendant la guerre de Cent Ans, c'est ce cadre spatial et chronologique qui a été retenu par la majorité des auteurs. En effet, parmi les 20 contributions qui composent ce recueil, seul un petit nombre est consacré à une aire géographique différente du royaume de France, cadre de $75 \%$ de l'ensemble ; les $25 \%$ restant traitent de l'Empire, de l'Angleterre, du royaume de Navarre, de la Bohême ou de l'Empire carolingien. La période chronologique indiquée en titre $\left(\mathrm{VIII}^{\mathrm{e}}-\mathrm{XV}^{\mathrm{e}}\right.$ siècle) est un peu trompeuse dans la mesure où une contribution seulement, celle de Christiane Veyrard-Cosme ( Réflexion politique et pratique du pouvoir dans l'œuvre d'Alcuin », p. 401-425), est consacrée au VIII ${ }^{\mathrm{e}}$ siècle. Sur les 19 contributions restantes, 6 (soit $30 \%$ de l'ensemble) concernent spécifiquement le XIV e siècle, 8 (soit $40 \%$ ) exclusivement le $\mathrm{XV}^{\mathrm{e}}$ siècle, 3 (soit $15 \%$ ) les deux derniers siècles du Moyen Âge, tandis que celles de Laurence Harf-Lancner et de Philippe Ménard (soit $10 \%$ ) dépassent le bas Moyen Âge, tout en l'englobant.

Spécialiste de la prosopographie historique, Autrand a d'abord dirigé ses recherches vers l'étude sociale des hommes gravitant dans les sphères du pouvoir, leur milieu et leur culture. Plus récemment, elle s'est intéressée à l'analyse de la littérature politique, qu'il s'agisse de traités savants, poétiques, ou de chroniques. Ces directions, et parfois ces méthodes, sont fidèlement reprises dans ces mélanges, et c'est l'attention particulière accordée au plus récent de ces thèmes de recherche qui confère à ce recueil - à rapprocher de celui dirigé par Nicole Pons et Monique Ornato, Pratiques de la culture écrite en France au XVe siècle (Louvain-la-Neuve, Fédération internationale des instituts d'études médiévales, 1995), auquel ont contribué Autrand et quelques auteurs présents dans ce volume - une unité et une cohérence remarquables : environ trois quarts des contributions ont en effet pour point de départ des sources littéraires à caractère politique, ou en examinent l'influence dans la pratique du pouvoir, comme le font celles des Paravicini ( « L'arsenal intellectuel d'un homme de pouvoir. Les livres de Guillaume Hugonet, chancelier de Bourgogne », p. 261-325), de Veyrard-Cosmes et de Lewis (« Pouvoir, "spéculative" et pratique : quelles voix entendre ?», p. 157-169). L'article de Ménard («Les conflits de pouvoir dans les fabliaux», p. 171-179) qui analyse les fabliaux est une exception dans ces quelque $75 \%$, tandis que Le Jugement du roi de Navarre de Guillaume de Machaut, étudié par Boutet («L'éloge du prince et l'expérience de la mélancolie. Réflexion sur les facteurs de cohérence du Jugement du roi de Navarre de Guillaume de Machaut», p. 33-45), est avant tout une œuvre poétique. Seuls l'article de Gauvard («Les juges jugent-ils ? Les peines prononcées par le Parlement criminel vers 1380-vers 1435 », p. 69-87) et les études 
prosopographiques d'Hélène Millet («Biographie d'un évêque rescapé de la méthode prosopographique. Jean de Sains, officier des ducs d'Anjou et secrétaire de Charles VI », p. 181-209), Jean-Philippe Genet (« Prosopographie et histoire culturelle. Origine sociale des auteurs médiévaux anglais », p. 89-109) et Jacques Paviot («Jacques de Luxembourg. Politique et culture chez un grand seigneur du $\mathrm{XV}^{\mathrm{e}}$ siècle », p. 327-341) n'utilisent pas les sources littéraires comme point de départ.

Cette unité n'est pas incompatible avec la grande diversité qui caractérise ces mélanges et qui en est l'un des points forts - diversité qui se manifeste aussi bien dans le type de sources littéraires étudiées (miroirs, suppliques, harangues, fables, poésies, traités politiques, chroniques), le choix des hommes (théologiens et théoriciens comme Jean Gerson, conseillers comme Smil Flaška, hommes de pouvoir comme Guillaume Hugonet, hommes de terrains comme Jean de Bueil...) que par les méthodes employées, les buts scientifiques poursuivis, et les approches choisies par les différents auteurs. À cet égard, l'association comme maîtres d'œuvre de cet ouvrage d'un professeur de littérature, Boutet, et d'un historien, Verger, a sans doute été décisive. Élisabeth Gaucher («Écriture de soi, écriture du politique : le Jouvencel », p. 55-67), par exemple, utilise la théorie littéraire dans son analyse du Jouvencel - livre de tactique militaire et de souvenirs de Jean de Bueil, dans lequel cet ancien conseiller de Charles VII, disgracié au début du règne de Louis XI, propose aux jeunes générations un modèle du bon chevalier. Cette utilisation permet d'une part d'apporter un regard nouveau sur ce texte maintes fois commenté par les historiens, et d'autre part de décoder cette autobiographie un peu différemment en montrant qu'elle est l'occasion de réactiver le débat sur l'individu face au pouvoir de l'État, et sur les relations entre prérogatives civiles et militaires. L'historienne Nicole Pons montre quant à elle tout aussi finement l'évolution de l'utilisation du thème de la vérité dans la littérature de propagande de guerre, mais d'une manière sans doute plus familière aux historiens («Pour ce que manifestation de Vérité. Un thème du débat politique sous Charles VI », p. 343-363). C'est aussi le cas de Jean-Marie Moeglin lorsqu'il étudie les stratégies discursives développées par les partisans de Henri VII pour établir la norme de l'empereur juste et inflexible selon laquelle il n'avait pas su se conduire à Crémone, et celle d'un empereur ayant choisi d'être miséricordieux à Brescia ( Henri VII et l'honneur de la majesté impériale. Les redditions de Crémone et de Brescia (1311) », p. 211-247).

Les buts poursuivis par les auteurs, ainsi que les méthodes employées divergent également. Certains établissent des éditions scientifiques de discours (c'est le cas, par exemple, de la «supplique à Charles VII pour que, de sa grâce, il allège les impôts de Lyon et du Lyonnais » par Contamine), ou d'inventaire de bibliothèque (celui notamment du chancelier Hugonet par les Paravicini qui font suivre cette édition d'une riche bibliographie) ; d'autres écrivent des articles dont il faut saluer le caractère didactique. C'est le cas de deux spécialistes de la prosopographie historique, Millet dans sa contribution sur Jean de Sains, et Genet qui traite de l'origine sociale des auteurs médiévaux anglais. Millet détaille sa méthode, décrit les étapes successives de sa recherche en indiquant les passages d'une source à une 
autre lorsque la précédente a atteint ses limites. Elle explique également les raisons pour lesquelles elle a choisi d'inclure l'évêque de Gap dans son étude des ecclésiastiques français ayant participé à la résolution du Grand Schisme, alors même qu'il ne figurait pas sur la liste des membres du concile de Pise de 1409. Genet pour sa part montre comment contourner les difficultés en utilisant des variables a priori peu exploitables comme le facteur « origine sociale inconnue », lorsqu'il s'agit de mener l'étude prosopographique d'un groupe. Ces deux excellents articles, complétés par des schémas et des tableaux, constituent en fait une véritable leçon pour les historiens peu familiers de la méthode prosopographique.

Outre la diversité des approches et des méthodes proposées par les auteurs, ce n'est pas le moindre de leur mérite que de proposer des analyses ou des pistes nouvelles sur des thèmes pourtant abondamment étudiés. C'est notamment le cas de Harf-Lancner («Les membres et l'Estomac. La fable et son interprétation politique au Moyen Âge », p. 111-125), qui parvient à produire un texte original sur l'image organique de la société humaine et son évolution, sujet classique s'il en est, en prenant pour point de départ la fable des membres et de l'estomac, ce qui est plus inhabituel. L'auteur montre en effet de façon magistrale comment on a adapté cette fable au contexte médiéval en lui redonnant sa signification politique, et les raisons pour lesquelles la métaphore du corps humain dans son entier lui fut préférée au Moyen Âge pour représenter la place du roi et de l'individu dans la société, alors restituée dans toute sa complexité. D'autres auteurs, à défaut de renouveler des analyses classiques, proposent néanmoins des pistes de recherche comme c'est le cas pour Martin Nejedlý («L'idéal du roi en Bohême à la fin du XVI e siècle. Remarque sur Le Nouveau Conseil de Smil Flaška de Pardubice », p. 247-259) qui, écrivant sur la Bohême, suggère à la fin de son article sur Le Nouveau Conseil de Smil Flaška, de relancer les études comparatives internationales et interdisciplinaires sur la « littérature didactique ».

Malgré ces nombreux points forts, quelques faiblesses sont néanmoins à déplorer. Ainsi, la contribution de Ménard qui traite des conflits de pouvoir dans les fabliaux est inattendue et aurait pu apporter une certaine originalité au recueil puisque l'auteur quitte la sphère du pouvoir politique pour entrer dans celle du privé, du ménage et des rapports de force entre conjoints. Malheureusement, on saisit peu la différence entre la deuxième partie ( $\mathrm{La}$ rébellion féminine ») et la troisième («Le monde renversé »), tandis que la conclusion finale nous « révèle » qu'un profond antiféminisme caractérisait ces textes et que leurs auteurs considéraient, comme l'Église, que le mari devait commander, et la femme obéir ; selon Ménard, « la société médiévale ne pouvait accepter l'égalité au sein du couple. Les revendications féminines en vue d'exercer le commandement paraissaient choquantes. Aujourd'hui les temps ont changé » (voir p. 180). Cette conclusion ne semble guère nouvelle...

Le recueil souffre parfois d'un manque de références bibliographiques. C'est particulièrement le cas en ce qui concerne les miroirs, pourtant étudiés dans cinq contributions (voir les articles d'Élisabeth Gaucher, Martin Nejedlý, Albert Rigaudière, Armand Strubel et Jacques Verger). Certes, Jacques Krynen, dont on aurait d'ailleurs bien voulu qu'il participât à l'élaboration de ces mélanges, est cité presque 
systématiquement, mais à part ses ouvrages (en particulier Idéal du prince et pouvoir royal en France à la fin du Moyen Âge (1380-1440). Étude de la littérature politique du temps, Paris, Picard, 1981), ceux de Dora M. Bell (L'Idéal éthique de la royauté en France au Moyen Âge d'après quelques moralistes de ce temps, Genève-Paris, Droz, 1962), et de Michel Senellart sur ce sujet (Les Arts de gouverner. Du regimen médiéval au concept de gouvernement, Paris, Le Seuil, 1995), la bibliographie reste sur ce point très succincte. De même, malgré la qualité de sa contribution, Élisabeth Gaucher ne cite aucune référence sur le Jouvencel. C'est également le cas de l'article par ailleurs fort intéressant d'Armand Strubel ( Le "chevauchier" de Charles V. Christine de Pizan et le spectacle de la majesté royale, p. 385-399), dans lequel on cherchera en vain la mention des ouvrages ou des articles principaux sur la «théatralisation du pouvoir» dont il parle (p. 386). En effet, alors qu'il examine cette question de la « ritualisation de plus en plus poussée de la vie publique » (ibid.), il ne cite pas l'ouvrage de Bernard Guenée et Françoise Lehoux (Les Entrées royales françaises de 1328 à 1515, Paris, CNRS, 1968) qui aurait pourtant eu sa place, en particulier dans la dernière partie de l'article intitulé «La Demonstrance de l'autorité royale». Enfin, on pourra déplorer que dans un recueil dont le titre est Penser le pouvoir, aucun article n'aborde l'histoire diplomatique pour laquelle Autrand a pourtant manifesté un intérêt certain (voir entre autres sa contribution dans Lucien Bély, dir., L'Invention de la diplomatie, Paris, Presses universitaires de France, 1998) et dont elle a démontré qu'elle était indissociable de l'élaboration des grandes théories politiques de la fin du Moyen Âge, en particulier avec «Le concept de souveraineté dans la construction de l'État en France, $\mathrm{XIII}^{\mathrm{e}}-\mathrm{XV}^{\mathrm{e}}$ siècle » (dans Serge Berstein et Pierre Milza, Axes et méthodes de l'histoire politique, Paris, Presses universitaires de France, 1998). Elle y souligne en effet (comme, entre autres, Randall Lesaffer avec « The concepts of war and peace in the $\mathrm{XV}^{\text {th }}$ century treaties of Arras », dans Denis Clauzel, Charles Giry-Deloison et Christophe Leduc, éd., Arras et la diplomatie européenne. $X V^{e-X V I}$ siècles, Arras, Artois Presses université, 1999, p. 165-182) que les instructions données aux ambassadeurs étaient aussi l'occasion, au même titre que les traités politiques comme le Songe du vergier d'Evrart de Trémaugon, de «fixer» les droits royaux et en particulier les droits de souveraineté. Il est donc dommage que cette piste importante n'ait pas été exploitée ici.

Malgré ces quelques failles, ces mélanges constituent un recueil de qualité et constituent une référence dans le domaine de l'histoire politique que Françoise Autrand a tant contribué à faire avancer et dont elle a en partie provoqué le renouveau historiographique. La majorité des contributions est digne de cette grande chercheuse et se situe dans la droite ligne de ses thèmes de recherche et des méthodes dont elle est spécialiste, tout en apportant parfois des regards différents, en ouvrant de nouvelles pistes de recherche, et en renouvelant des analyses dont on aurait presque pu penser qu'elles étaient définitives. 
Au cloître et dans le monde. Femmes, hommes et sociétés (IXe-XVe siècle). Mélanges en l'honneur de Paulette L'Hermite-LeclercQ. Textes réunis par Patrick HenRIET et Anne-Marie Legras. Paris, Presses de l'université de Paris-Sorbonne, 2000. $18 \times 23,5,396$ p., bibliogr. (Cultures et civilisations médiévales, XXIII).

Au cloître et dans le monde est un bouquet de 31 contributions offertes à Paulette l'Hermite-Leclercq à l'occasion de son départ à la retraite. Coordonnés par Patrick Henriet et Anne-Marie Legras, ces mélanges sont organisés en cinq grandes parties qui font la part belle, mais non exclusive, aux dames et à leur place dans la société médiévale.

La première partie, «Regards masculins sur la femme», commence par l'essentiel, en soulignant le poids des sources. La plume des clercs fait autorité. L'exégèse biblique élabore des systèmes de valeurs complexes comme le montre Gilbert Dahan au travers des interprétations du verset «nigra sum sed formosa » du Cantique des cantiques. Le discours masculin, traditionnellement misogyne, est capable de nuances. Les exempla des sermons de Robert de Sorbon présentés par Nicole Bériou mettent en garde contre la femme tentatrice et valorisent la mère aimante ou la pieuse béguine. Toutefois ces images utilisées pour affermir la foi des clercs et des laïcs n'altèrent pas le principe qui fait de la femme une fille d'Ève soumise à l'homme. On aurait pu croire que l'essor du culte marial à la fin du $\mathrm{XI}^{\mathrm{e}}$ siècle le remettrait en cause, l'exaltation de la Vierge tirant vers le haut ses « sœurs en humanité » (p. 52). Rien n'est moins sûr comme le montre Marielle Lamy par l'étude d'un ouvrage de mariologie de la fin du XIII ${ }^{\mathrm{e}}$ siècle attribué à Albert le Grand. En Syrie, les auteurs ayyoubides ont chanté les louanges de régentes, de femmes soldats et plus couramment d'érudites ou de dévotes. Mais comme le souligne Anne-Marie Eddé, la régente Dayfa Khatun ou les sœurs de Saladin sont des privilégiées et les récits transmettent aussi une autre image, plus traditionnelle, qui fait de la femme un «être fragile » (p. 76). Ici comme ailleurs, l'homme reste son meilleur gardien. Pour Philippe de Mézières qui conçoit l'ordre de la chevalerie de la passion de Jésus-Christ, la présence d'une épouse aux côtés du chevalier aidera au succès de la reconquête. Exposées par Philippe Contamine, les règles de l'ordre gèrent dans le détail le comportement en Terre sainte de ces « religieuses mariées » (p. 83).

La deuxième partie aborde un thème central des travaux de L'Hermite-Leclercq : le monachisme. "Vivre en communauté » oui, mais sous quelle règle ? Michel Parisse rapporte le cas du jeune manceau Rigran qui choisit de troquer la coule et l'austère vie monastique contre l'aube et les facilités de la vie canoniale à la fin du $\mathrm{IX}^{\mathrm{e}}$ siècle. L'auteur anonyme du récit, sans doute moine, n'a que mépris pour l'engagement final de Rigran sur cette «voie de perdition» (p. 92). Considérée comme supérieure, la vie monastique connaît un renouveau important avec la fondation de Cluny. Le succès de l'abbaye bourguignonne se mesure dans des sources, présentées par Dominique Iogna-Prat, qui célèbrent la mémoire clunisienne et construisent le «miroir du passé » de l'ordre (p. 103). Cluny veille à la pureté de la vie claustrale. Isabelle Cochelin s'intéresse à un aspect peu connu de la discipline clunisienne concernant certains moines, les iuniores sub custodia âgés 
de quinze ans et placés chacun sous la surveillance d'un gardien, le temps de passer le cap dangereux de l'adolescence et de faire l'apprentissage définitif de la virginité. Sur le long terme, le projet clunisien s'essouffle ou parfois « s'enlise » (p. 142) comme le montre Denyse Riche dans son étude du réseau des prieurés en Provence du $\mathrm{X}^{\mathrm{e}}$ au XV $\mathrm{XV}^{\mathrm{e}}$ siècle. L'intérêt des mélanges se porte aussi sur d'autres ordres, plus petits comme celui de Grandmont présenté par Dom Jean Becquet à la fin du Moyen Âge, ou dont les innovations ne doivent pas être négligées. L'intéressant article d'André Vauchez mesure justement l'apport des ordres religieux à la notion de démocratie. Contrairement à la stricte pyramide clunisienne, Cîteaux et les Frères Mendiants ont privilégié une répartition plus équilibrée de l'autorité au sein de leurs ordres : le chapitre général, le recours à l'élection, le scrutin à bulletins secrets sont des jalons importants dans la genèse du régime représentatif en Occident. Le monastère est bel et bien un acteur de la vie de la cité et comme l'indique Élisabeth Crouzet-Pavan avec l'exemple de Venise, il peut jouer un rôle symbolique fort dans la géographie urbaine. Ponctuée par des «monastères sentinelles » (p. 162) qui délimitent et protègent l'espace vénitien, la ville apparaît comme la cité élue de Dieu. L'exemple florentin développé par Christiane Klapisch-Zuber rappelle enfin que les couvents ont une fonction sociale pour l'élite qui y place plus volontiers ses filles que ses fils.

Cantonnée de préférence à la sphère privée, la femme médiévale se distingue peu ou de façon assez convenue. La troisième partie, «Femmes laïques, entre pouvoirs et dévotions », expose toutefois trois cas d'expression manifeste d'un pouvoir féminin. Jean-Claude Cheynet s'intéresse aux rares dames qui ont reçu à Byzance une dignité éminente manifestée par le port du lôros. Ceignant cette somptueuse écharpe, la patricienne à ceinture ou zôstè n'est devancée à la cour que par l'impératrice. Dans la péninsule ibérique, certaines infantes, filles ou sœurs de roi, célibataires, reçoivent l'infantado impliquant la protection de puissants monastères, et participent ainsi à une sorte de sanctification du pouvoir monarchique. L'institution décrite par Patrick Henriet culmine avec Sancha, sœur d'Alphonse VII, et le monastère de Saint-Isidore de León. Enfin Frédérique Lachaud envisage les dépenses vestimentaires de Philippa de Hainaut pendant l'année 1332-1333 comme un élément important de la politique de prestige voulue par le roi Édouard III et un moyen pour la reine de construire autour d'elle un solide réseau de fidélités. Mais Sancha ou Philippa sont des cas exceptionnels. La majorité des dames médiévales se caractérise par des pratiques pieuses plus ou moins ferventes, qu'il s'agisse des Toscanes du contado florentin décrites par Charles-Marie de La Roncière ou des Hongroises moins connues présentées par Marie-Madeleine de Cevins. Parfois les testaments ou les inventaires permettent de dégager plus précisément les dévotions et les goûts de l'une d'entre elles. Bertrand Schnerb puis Jacques Paviot donnent deux exemples emblématiques d'une noblesse pieuse et cultivée : Marguerite de Bécourt, dame de Santes et Jeanne de Chalon, comtesse de Tonnerre.

Abordant un autre type de sources, la quatrième partie « Droit, femmes, justice » traite du thème du conflit. La fragilité sociale des femmes apparaît clairement au travers de quelques lettres de rémission données en exemple par Annie Saunier. Les archives de l'Échiquier de Normandie, dépouillées par Henri Dubois, témoignent 
de dames nobles seules qui défendent en appel des affaires typiquement féminines, comme la dot et le douaire, ou des questions d'héritage et de patronage d'église. L'exemple forézien d'Haynarde le Sourd développé par Romain Telliez montre de façon originale la difficulté de faire valoir les droits de la femme en cas d'annulation du mariage. Mais au-delà de la discrimination sexuelle qu'il illustre, le conflit entraîne une perturbation du fonctionnement normal de la société. Comment rétablir alors la concorde ? Par l'enquête qui détermine les droits de chacun comme l'expose Alain Provost dans l'affaire qui opposa l'évêque de Troyes à l'abbesse de Notre-Dame-aux-Nonnains au sujet du droit des moniales à garder le cheval du nouveau prélat qui accède à l'épiscopat. Les dépositions des témoins font alors de la parole, non sans biais, le moyeu du processus informatif. Quand elle est pervertie, la parole fait également l'objet d'une répression. C'est le cas du blasphème exploré par Corinne Leveleux-Teixeira dans une étude ambitieuse qui cherche derrière ce «scandale du discours » (p. 327) l'évolution du concept de vérité. Les sources hagiographiques font de la médiation religieuse un autre moyen de concorde. Dans la Flandre des $\mathrm{XI}^{\mathrm{e}}$ et $\mathrm{XII}^{\mathrm{e}}$ siècles, l'action pacificatrice de saint Arnoul est décodée par Dominique Barthélemy avec l'aide ponctuelle du « faisceau lumineux » de l'anthropologie (p. 272).

Une cinquième partie intitulée « Rites, liturgie, spiritualité » clôt les mélanges. Qu'il s'agisse de l'évangile de la messe étudié par Pierre-Marie Gy ou des prescriptions synodales attentives à la dévotion eucharistique cataloguées par Jean Longère, la liturgie joue un rôle essentiel de structure du culte. Cette codification se retrouve dans les rituels consignés par les pontificaux. À partir des descriptions de la cérémonie de consécration des vierges, Catherine Vincent analyse l'emploi des cierges et du signe de lumière dans le cérémonial chrétien d'un point de vue historique et symbolique. Ces pratiques traduisent par des gestes et des processions certains temps forts de la communauté chrétienne. L'étude faite par Damien Boquet des sermons et traités ascétiques cisterciens laissés par Guerric d'Igny et Aelred de Rievaulx donne un aperçu de l'intense recherche spirituelle menée par ces deux « explorateurs de l'âme » et des émotions (p. 377). Enfin après les gestes et les discours, l'article de Daniel Russo apporte une pierre supplémentaire à l'édifice spirituel en envisageant le rôle de l'image religieuse à travers l'exemple de saint François d'Assise.

Que ce soit dans le cloître ou dans le monde, attentifs à la valeur des stéréotypes et des originalités, ces articles dressent quelques portraits féminins et masculins de la société médiévale et mettent à l'honneur des thèmes chers à Paulette L'HermiteLeclercq, auteur du Monachisme féminin dans la société de son temps (Paris, Cujas, 1989) et de L'Église et les femmes dans l'Occident chrétien des origines à la fin du Moyen Âge (Turnhout, Brepols, 1997). 
Anne-Marie Flambard HÉRICHER, dir., Frédéric II (1194-1250) et l'héritage normand de Sicile. Colloque de Cerisy-la-Salle, 25-28 sept. 1997. Caen, Presses universitaires de Caen, 2000. $16 \times 24$, 240 p., ill., bibliogr.

Certains secteurs de l'histoire médiévale attirent plus que d'autres, parce qu'ils sont fortement liés à l'imaginaire collectif. Ainsi, l'histoire du XIII ${ }^{\mathrm{e}}$ siècle, qu'on a pu dire ces dernières années la parente pauvre de l'historiographie médiévale, par contraste avec le renouveau de l'histoire du Moyen Âge central (XI ${ }^{\mathrm{e}}-\mathrm{XII}^{\mathrm{e}}$ siècle) et l'explosion des études sur le bas Moyen Âge, voit pourtant les publications sur deux de ses figures les plus illustres se renouveler constamment. À la figure charismatique du roi de France Saint Louis, naguère remise en lumière par Jacques Le Goff (Saint Louis, Gallimard, 1996), correspond pour les Italiens et les Allemands celle de l'empereur germanique et roi de Sicile Frédéric II Hohenstaufen, son contemporain (né en 1194, roi de Sicile en 1197, empereur du Saint Empire romain germanique couronné en 1220). Comme Saint Louis, Frédéric II bénéficie du privilège historique douteux de concentrer en lui l'intérêt de la recherche proprement dite et un engouement plus large, dû à sa stature quasi mythique dans la construction historiographique nationale allemande et italienne, et à son rôle de figure tutélaire du Mezzogiorno. La figure complexe de l'empereur, héritier des prétentions impériales du Saint Empire et du royaume méditerranéen, à l'origine à moitié musulman, des Normands de Sicile, ouvert aux spéculations scientifiques les plus audacieuses du temps (l'averroïsme) et restaurateur malheureux de la puissance impériale en Italie du Nord, explique en grande partie cette attirance. La place exceptionnelle tenue, plus de quatre-vingt ans après sa rédaction, par l'ouvrage mi-historique mi-littéraire L'Empereur Frédéric II d'Ernst Kantorowicz illustre bien le caractère ambigu de cette fascination, qui n'est pas sans poser divers problèmes aux historiens professionnels.

Les années 1994-1997, dates anniversaires de la naissance et de l'accession au trône sicilien de Frédéric II, ont ainsi vu déferler colloques et célébrations en Italie surtout, mais aussi en Allemagne. Dans cet ensemble qui a permis de faire le point sur l'état de la recherche sur Frédéric II et ses royaumes, la France fait un peu figure, pour des raisons historiques, de parent pauvre. L'histoire de l'Italie souabe (les Hohenstaufen règnent en Sicile de 1191 à 1266) est une spécialité largement dominée par la recherche allemande. La parution du colloque de Cerisy-la-Salle de 1997, Frédéric II (1194-1250) et l'héritage normand de Sicile, dirigé par AnneMarie Flambard Héricher, vient en partie combler cette lacune, en proposant une perspective française sur le règne de Frédéric II, héritier des rois normands par sa mère.

Le volume s'ouvre par une introduction en deux partie. La première consiste en un résumé événementiel de la vie mouvementée de l'empereur, sous la plume de Flambard Héricher ( "Du "gamin d'Apulie" à la "splendeur du monde", les grandes étapes du règne de Frédéric II », p. 15-28), qui rend bien compte des différentes phases d'une vie dont les inflexions les plus décisives sont l'accession au trône impérial en 1212, confirmée par le couronnement de 1220, qui fait du jeune roi de Sicile à l'autorité disputée le personnage le plus puissant de la chrétienté, et la mise 
au ban de la chrétienté à travers l'excommunication de 1239 et la déposition de l'empereur au concile de Lyon I (1245), qui marque l'ultime échec d'une conciliation entre les Hohenstaufen et le pouvoir papal. La seule fausse note est le lapsus calami page 24, où il faut rétablir Mayence à la place de Manzona, au sujet de la diète de Mayence. La seconde contribution, originale, de Jean-Marie Martin («Quelques réflexions sur Frédéric II et la France », p. 29-49), commence par un parallèle historiographique des figures de Frédéric II et Saint Louis, s’interroge sur la diversité des espaces gouvernés en droit par l'empereur (Germanie, Italie du Nord, Italie du Sud, royaume d'Arles au sud-est de la France actuelle, royaume latin de Jérusalem), pour finir par un point sur les lacunes et les antécédents de l'historiographie proprement française sur Frédéric II. L'auteur, un des rares spécialistes français de l'Italie du Sud, souligne les lacunes de cette historiographie, par opposition à des débuts brillants au XIX ${ }^{\mathrm{e}}$ siècle.

La seconde grande division du colloque («Frédéric II et son temps ») regroupe trois contributions. Errico Cuozzo («Frédéric II et le Mezzogiorno », p. 53-62) donne, dans une synthèse rapide, quelques éléments sur les liens du règne avec l'histoire sociale et intellectuelle des deux parties de l'Italie; le grand savant allemand Norbert Kamp, dont il s'agit ici d'un de ses derniers articles publiés, débrouille l'écheveau des relations complexes entre le pouvoir politique de Frédéric II et les églises du royaume de Sicile ( «L'héritage normand dans la politique ecclésiastique de Frédéric II », p. 63-78), tandis que le spécialiste français de l'histoire sicilienne par excellence qu'est Henri Bresc fait le point sur les relations entre le monde musulman - à l'intérieur et à l'extérieur de ses royaumes - et Frédéric II ( « Frédéric II et l'Islam », p. 79-92). Elles sont éminemment paradoxales, puisque la répression politique des derniers ferments d'indépendance sarrasine en Sicile, et la déportation des populations musulmanes du royaume à Lucera, en Italie continentale, n'empêchent pas une valorisation extrême, à la cour, des échanges avec les grands souverains du monde musulman, et une volonté de connaissance de la grande culture islamique du temps.

Une troisième partie regroupe quelque peu artificiellement «Vie économique et culture ». Jean-Marie Martin livre une seconde contribution, cette fois en tant que spécialiste de l'économie sud-italienne et des Pouilles («Problèmes économiques à l'époque de Frédéric II », p. 95-107), sur les problèmes posés à l'historien par les tentatives étatiques pour contrôler l'économie du royaume. Lucia Travaini fait le point sur la politique monétaire fort novatrice de Frédéric II («La monnaie à l'époque de Frédéric II », p. 115-128). C'est également à un des symboles majeurs de l' «inflexion frédéricienne » que s'attaque Maylis Baylé dans «L'évocation de l'antiquité dans l'art de l'Italie méridionale au temps de Frédéric II » (p. 131-146). Enfin Salvatore Fodale présente une brève synthèse sur les intérêts scientifiques de l'empereur («Frédéric II savant et empereur », p. 147-156).

La partie suivante, « Châteaux et pouvoir », est plus proprement archéologique et architecturale. Flambard Héricher livre une riche présentation de la politique générale de construction de Frédéric II dans le royaume de Sicile («Les châteaux du royaume de Sicile au temps de Frédéric II », p. 159-198), tandis que Patrice 
Beck analyse de manière détaillée un cas architectural particulier (« Castel Fiorentino en Capitanate, domus de Frédéric II », p. 199-212).

Enfin, une partie composée d'un unique article sert en quelque sorte de conclusion («Postérité »). Marina Marietti y donne une analyse littéraire des « Héritiers des Normands dans La Divine Comédie de Dante » (p. 215-227). L'ensemble est complété par une bibliographie assez riche, rendue néanmoins obsolète par celle, beaucoup plus exhaustive de la nouvelle biographie de Frédéric II en langue allemande Friedrich II, de Wolfgang Stürner, parue à Darmstadt (Nimbus Verlag), en deux volumes, respectivement en 1992 et 2000.

Que conclure de ce colloque ? Il offre les mêmes qualités et les mêmes défauts que la plupart des colloques internationaux sur Frédéric II qui se sont succédé entre 1994 et 1997, et dont il est un des derniers parus. Certains articles sont novateurs dans leurs perspectives, richement documentés, et font référence à la bibliographie la plus récente (c'est notamment le cas des contributions de Bresc et Martin, et des articles techniques sur les constructions en Sicile de Flambard Héricher et Beck). En revanche, on est assez désagréablement frappé par le caractère extrêmement synthétique et, il faut bien le dire, répétitif d'une partie des contributions dont les renvois bibliographiques sont extrêmement datés, et qui tiennent plus du simple essai que de l'article de recherche (on pense en particulier à Fodale et Marietti). Il y a peut-être là un effet pervers dû au statut de « puissance invitée » de la recherche italienne, alors que des chercheurs français, en situation d'infériorité numérique face à la riche historiographie allemande et italienne, ont tenu à apporter du nouveau. On peut y voir aussi plus prosaïquement une tendance due à l'enfermement d'historiographies nationales dans leurs champs respectifs. Les travaux les plus novateurs dans bien des secteurs de la recherche sur Frédéric II ont été rédigés en allemand, langue que ne lisent pas forcément les chercheurs italiens et français, comme leurs références l'enseignent. En général, en dépit de l'internationalisation de la discipline dont les colloques sont censés être le reflet, les difficultés de circulation des données entre les différents pays sont perceptibles, particulièrement quand il s'agit de renouveler l'histoire d'une époque, le règne de Frédéric II, qui est, pour bien des amateurs d'histoire, mais aussi des historiens, véritablement mythique.

Benoît GRÉVIN

Anne-Marie Lamarrigue, Bernard Gui (1261-1331). Un historien et sa méthode. Paris, Honoré Champion, 2000. $15 \times 22,528$ p., sources, bibliogr., index (Études d'histoire médiévale, 5).

Il y a maintenant vingt ans, Bernard Guenée, dans son Histoire et culture historique dans l'Occident médiéval (Paris, Aubier Montaigne, 1980), bouleversait les cadres bien établis de l'étude de l'historiographie médiévale. La « révolution » opérée n'en était pas vraiment une, dans une optique anthropologique un peu élargie : il 
s'agissait avant tout de rendre les travaux des historiens médiévaux à la logique propre d'une civilisation de la compilation, où l'histoire s'inscrit dans le champ du religieux et de la mémoire et non dans une perspective littéraire ou scientifique, d'une civilisation où l'individu ne s'affirme pas encore, et notamment, pas encore littérairement, en dehors du groupe. Ce renversement de perspective, qui paraît aujourd'hui évident, était encore largement nécessaire, tant la vision des historiens médiévaux était empreinte des clichés amoncelés par les historiographies nationalistes et romanesques du XIX et du début du XX $\mathrm{XX}^{\mathrm{e}}$ siècle. Pour opérer ce changement de perspective, il fallait donc appréhender le métier de l'historien médiéval en jugeant ses productions non à l'aune de nos critères historiques propres mais de ses outils, en particulier la technique de la compilation, et de sa vision du monde.

L'impulsion donnée par Guenée s'est concrétisée dans de nombreux travaux, dont ceux de Colette Beaune sur le développement du sentiment historique et national en France (Naissance de la nation France, Paris, Gallimard, 1985) et d'AnneMarie Lamarrigue, concernant l'historiographie médiévale et tout particulièrement le personnage et les écrits d'un clerc très représentatif de la culture historique moyenne en France méridionale au tournant des XIII et XIV ${ }^{\mathrm{e}}$ siècles, Bernard Gui. Bernard Gui (1261-1331), un historien et sa méthode, présente le résultat de ces travaux.

Ce livre se veut une application de ces nouvelles approches historiques à l'étude de l'historiographie médiévale à partir du cas à la fois particulier et représentatif d'un historien prolifique, justement choisi pour sa représentativité et son adéquation aux clichés de l' «historien médiéval médiocre » cher aux auteurs positivistes. L'œuvre de Bernard Gui, qui consiste en de nombreuses chroniques, allant de l'histoire universelle à l'histoire locale (chronique des rois de France, chronique des comtes de Toulouse, traité sur l'histoire du monastère Saint-Augustin de Limoges, catalogue des évêques de Limoges, de Toulouse, traité des saints du Limousin, chronique des prieurs de Grandmont et de l'Artige...), peut apparaître à première lecture comme porteuse de tous les éléments qui déparent dans une optique moderne le genre de l'histoire médiévale. Absence apparente de perspective proprement historique claire, surabondance de la compilation, localisme sont trois des éléments qui pourraient la caractériser. En restituant minutieusement la logique de l'historien médiéval, Lamarrigue parvient justement - c'est tout l'intérêt de la démarche initiée par Guenée - à montrer que le processus de compilation et d'organisation des matériaux possède une logique propre, qui n'est pas celle de l'historien moderne, mais qui, dans un cadre conceptuel très différent du nôtre, obéit à des règles strictes. Le respect de cette démarche, démarche historique, mais dans un sens évidemment différent de celui que ce terme a actuellement, fait de Bernard Gui un bon historien, dans l'optique, non créative, et non proprement scientifique, de l'histoire médiévale. Il faut dire que l'auteur a un parti pris bien naturel de sympathie, voire de relative empathie avec l'historien Bernard Gui, qui l'amène peut-être à mettre en avant les qualités d'historien de ce dernier, nous y reviendrons.

Conformément aux prémisses de sa méthode, Lamarrigue a organisé son analyse en quatre sections, respectant le travail de l'historien. Elle présente d'abord 
dans une longue introduction (p. 37-59) l'homme Bernard Gui. Celui-ci, tôt entré dans l'ordre dominicain, se distingua principalement par ses activités d'inquisiteur dans un grand Sud-Ouest entre Languedoc et Limousin, tout en menant de front une carrière relativement effacée d'administrateur dominicain et son œuvre, abondante, d'historien. Comme on l'aura compris à l'énoncé des chroniques, traités et catalogue cités plus haut, l'histoire n'est pas dissociable chez ce religieux d'un enracinement très profond dans les réalités d'une Église aux assises locales.

Les quatre parties de l'ouvrage permettent, dans une succession logique, de pénétrer dans l'atelier de l'historien médiéviste, en partant du matériau historique proprement dit, pour arriver au dégagement de la perspective historique.

La collecte et l'utilisation des sources (Première partie : «L'information de l'historien. Les sources », p. 63-168) fait apparaître un tableau très contrasté : à la dépendance très grande envers l'héritage dominicain ou les chroniques locales, aux différents obstacles techniques, s'oppose un véritable souci de recherche des documents originaux qui s'affirme au fil de la composition des différentes œuvres.

La deuxième partie («L'écriture de l'histoire », p. 171-268) analyse la méthode de rédaction utilisée par Bernard Gui. C'est peut-être la plus instructive, dans la mesure où l'auteur a poussé très avant l'analyse des techniques de compilation et d'abréviation utilisées par l'historien, et au cœur de la pratique de l'histoire médiévale. La mise en valeur d'un travail de confrontation des sources et d'un véritable exercice de critique historique, encore que ce dernier terme pose problème, comme on le verra en conclusion, clôt cette section.

La troisième partie assure la transition entre l'analyse des procédés d'écriture et celle de la vision historique développée par Bernard Gui («Les repères de l'histoire : temps et espace », p. 271-375). En effet, le temps et l'espace évoqués sont à la fois ceux de la datation et de la localisation de ses objets d'études par l'historien, enjeux d'un travail complexe sur les sources, et ceux de sa perception propre, banale, mais hautement révélatrice dans le cadre de l'étude de la vision médiévale de l'histoire. Elle révèle une focalisation extrême sur la région d'activité de l'historien, mais aussi la permanence de cadres conceptuels hérités (nomenclature antiquisante) révélatrice de la pesanteur des localisations et des traditions.

Enfin, la quatrième et dernière partie («Les perspectives de l'historien », p. 379-465) s'enchaîne naturellement avec la précédente, tout en développant les trois orientations principales de l'historien Bernard Gui : l'inscription de son récit historique dans un cadre méridional, celui d'une Aquitaine encore proche de la grande principauté carolingienne par son extension géographique, aux abords des profonds bouleversements du XIV siècle ; l'empreinte d'une appartenance à l'ordre des Dominicains et, au travers d'un soutien sans faille à l'Église, l'hostilité aux déviances religieuses, mais aussi à l'importance croissante du pouvoir laïque (exprimée, par exemple, par la lutte entre la papauté et Philippe le Bel, dont on perçoit particulièrement les échos dans ces chroniques d'ambiance méridionale) ; enfin, non sans quelque contradiction, l'intégration de ces deux éléments dans une vision de la monarchie française bien constituée qui met l'accent, à partir des origines mythiques (le mythe troyen), sur une continuité symbolisée par la « galerie des rois ». 
C'est sans doute cette dernière partie qui renferme, au-delà de la solidité de l'analyse, les développements les plus contestables. En effet, dans un ensemble de considérations très mesurées, il faut bien, faute de connaître les pensées intimes de Bernard Gui, se prononcer sur sa vision, sa perspective proprement historique, en tant qu'historien du midi, membre actif de l'Église et sujet du royaume de France. Dans l'ensemble, le tableau brossé par Lamarrigue est instructif et mesuré. Dans la tourmente d'un rattachement du Sud-Ouest au royaume qui n'en finit pas de laisser des traces, liées à l'hérésie et au démantèlement de l'ancienne principauté des comtes de Toulouse, un siècle après la croisade albigeoise, il faut faire la part entre le Bernard Gui inquisiteur et homme d'Église et le témoin du temps. Justement, la vision dans la perspective universalisante de l'Église l'emporte sur les considérations plus proprement régionales. Mais, dans un chapitre sur «L'éthique du chroniqueur » (p. 421-423), les formules rhétoriques employées par l'historien pour souligner les travers de ces éternels rivaux des Dominicains, auxquels appartient Bernard Gui, que sont les Franciscains, sont comprises au premier degré, comme des silences que le chroniqueur s'imposerait. Ces formules antiphrastiques (Bernard Gui affecte de se taire par honneur pour la religion...), sous leur couvert de pudeur, sont un tour pour attirer charitablement l'attention sur les débordements des éternels rivaux. On retrouve alors le problème du jugement personnel de l'historien contemporain quand, au-delà de la méthode, il a à examiner des passages empreints de subjectivité. $\mathrm{Si}$, dans le passage évoqué, nous croyons que Lamarrigue révèle une conception trop irénique des conflits au sein des ordres religieux au tournant des XIII ${ }^{\mathrm{e}}$-XIV ${ }^{\mathrm{e}}$ siècles, peut-être les meilleurs historiens positivistes ou romantiques si peu compréhensifs pour la pratique de l'historien médiéval auraient-ils, avec leur mauvais esprit habituel, au contraire souligné ces lignes de faille derrière l'apparence du discours pieux. C'est tout le danger d'une approche relativiste où l'empathie avec l'auteur ou la pensée étudiée doit parfois suppléer à une reconstitution jamais totalement réalisable, avec le risque de confusion inhérent à ce genre de rapprochements.

Un problème plus général, liée à la perspective relativiste implicitement à l'œuvre, est posé par l'affirmation d'un professionnalisme dans le métier d'historien lié notamment à la critique attentive des sources et aux tentatives de résolution des contradictions par Bernard Gui. On retrouve là la limite du renversement de perspective nécessaire pour pénétrer les logiques médiévales. Dans quelle mesure faut-il justement mettre en avant la cohérence et une certaine scientificité dans l'étude des documents historiques chez les historiens médiévaux ? Le problème se pose également à Gilbert Dahan dans son étude de l'approche de la Bible au Moyen Âge (L'Exégèse chrétienne de la Bible en Occident médiéval. XII ${ }^{e}$-XIV $V^{e}$ siècle, Paris, Éditions du Cerf, 1999). Il insiste avec raison sur les remarquables résultats de la critique textuelle de la Bible par les Dominicains et les Franciscains au XIII ${ }^{\mathrm{e}}$ siècle - résultats dignes de la critique scientifique qui s'affirmera quelques siècles plus tard. Mais n'y a-t-il pas une contradiction entre l'affirmation, sans cesse répétée, de la nécessité de retrouver les cadres conceptuels des médiévaux, leur logique propre, et la mise en avant positive de ceux de ces traits qui répondent le mieux aux critères de la logique historiographique moderne ? Ce point touche bien sûr au problème général des limites du relativisme en histoire, et ne saurait valoir comme 
critique de la démarche de Lamarrigue en particulier. C'est justement la qualité de sa démonstration, à travers le cas de Bernard Gui, qui permet, la lecture de l'ouvrage achevée, d'entamer une réflexion sur les nouveaux apports et les nouveaux problèmes soulevés par l'étude de l'historiographie médiévale.

Benoît GRÉVIN

Luca BianchI, Censure et liberté intellectuelle à l'Université de Paris, XIIIXIV siècles. Paris, Les Belles Lettres, 1999. $15 \times 21,5$, X-385 p., bibliogr., index (L'Âne d'or).

Le livre de Luca Bianchi est né d'un séminaire sur les condamnations universitaires à Paris aux XIII ${ }^{\mathrm{e}}$ et $\mathrm{XIV}^{\mathrm{e}}$ siècles, tenu à la Ve section de l'École pratique des hautes études en mai 1996, et ses deux premiers tiers ont connu une première élaboration sous la forme de deux longs articles. Le livre porte encore les traces de cette élaboration progressive : rarement un historien de la philosophie aura ouvert aussi large la porte de son «atelier». L'ouvrage se compose de trois parties très distinctes, ce qui n'est que partiellement reflété par le titre Censure et liberté intellectuelle à l'Université de Paris (XIII ${ }^{e}-X I V^{e}$ siècles), qui est aussi celui de la première partie (p. 19-85), restée très proche de l'article de 1996. Les deux parties suivantes reprennent le problème des censures et interdictions, non plus sous l'angle d'une analyse générale, mais sous celui de problèmes spécifiques, celui de la réception d'Aristote à Paris (1210-1366, p. 87-162), puis celui de la fameuse condamnation de 1277 (p. 163-230), et sont l'occasion de démonstrations nouvelles et très convaincantes sur des questions pourtant déjà largement abordées par l'historiographie.

L'introduction précise le cadre conceptuel de l'analyse, qu'on peut résumer en quatre points. Bianchi rappelle d'abord la diversité des condamnations au sein de l'Université médiévale, remarquant que ces nuances n'étaient pas toujours claires pour les contemporains eux-mêmes, et que l'étude des décisions institutionnelles doit s'accompagner d'une analyse des pratiques réelles du monde universitaire à l'égard des hommes et des doctrines stigmatisés. Deuxièmement, il souligne la nécessaire historicisation des débats doctrinaux au cœur des affaires de censure. Il ne s'agit pas d'un vœu pieux mais d'une mise en garde à l'égard d'un problème majeur de méthode en histoire intellectuelle : comment faire l'histoire d'un débat qui a été tranché, sans subir soi-même l'influence de la chose jugée, qui a tendance à figer orthodoxie et hétérodoxie dans une éternité factice ? Il est évident que les questions dogmatiques doivent nécessairement être mises à distance, le dogme luimême ne se définissant souvent qu'à partir de condamnations. Troisième aspect du problème, Bianchi affirme qu'une histoire des censures universitaires ne peut pas se passer d'une histoire du pouvoir qui contrôle le savoir. Il y a bien sûr les pouvoirs extérieurs au milieu universitaire, comme la hiérarchie ecclésiastique, à commencer par les évêques de Paris, comme Étienne Tempier. Mais l'Université elle-même constitue aussi un pouvoir paradoxal : créée comme une institution 
destinée à encadrer la production de savoir, elle aspire également à l'autonomie, non seulement institutionnelle mais intellectuelle, et finit par représenter un pôle de l'autonomisation du savoir face aux censures. Enfin, au sein de l'Université elle-même, on retrouve des enjeux de pouvoir, comme les luttes opposant les différentes facultés, ou les ordres religieux, ce qui a aussi des effets sur les querelles intellectuelles. Dernier point souligné par l'auteur, le problème de la constitution, en bonne partie sous la pression des pouvoirs évoqués à l'instant, de mécanismes d'autocensure, par lesquels les universitaires anticipent une condamnation qui ne manquerait pas de les frapper selon les critères qu'ils croient être ceux des censeurs. Bianchi a tout à fait raison de faire une place à cette dernière forme de limitation de la production intellectuelle, car elle est sans doute la moins étudiée et la moins facile à mettre en évidence, mais peut-être aussi la plus efficace, comme dans toute institution qui fonctionne bien.

Ces réflexions guident la recherche à travers les différents épisodes de censure et construisent une méthode qui conduit l'auteur à se situer par rapport à deux thèses relatives à la censure à l'Université. Celle de Charles H. Haskins dans The Rise of Universities (1923), tout d'abord, qui voyait l'Université médiévale comme un lieu de grande liberté, assez bien préservée des contraintes religieuses et politiques, dans laquelle le principe d'autorité était bien accepté, comme une règle du jeu scolastique en quelque sorte, et où les philosophes se sentaient libres du fait même de cette acceptation ( « une barrière n'est pas un obstacle pour ceux qui n'ont aucun désir d'aller au-dehors », comme le dit Haskins ), thèse dont Bianchi semble souvent éloigné. D'autre part, plus proche de sa propre position, celle d'Alain de Libéra dans Penser au Moyen Âge (Paris, Seuil, 1991), qui considère la censure comme un « opérateur historique » contribuant souvent à construire et fortifier les positions qu'elle attaque.

La première partie du livre analyse l'acte même de censure, en procédant à un essai typologique qui clarifie notablement les pratiques de l'Université médiévale. Les « condamnations » cachent une multiplicité de situations à ne pas mettre sur le même plan. Les censures sont classées suivant leur plus ou moins grande gravité, à l'intérieur de trois catégories, selon que les cibles soient des idées, des hommes ou des livres. Les livres peuvent être brûlés, sinon interdits, confisqués ou limités dans leur usage didactique, selon une logique décroissante, enfin font sans doute l'objet, loin en amont, d'une censure préalable, une censura praevia, avant même leur publication. Les idées subissent également des contraintes diverses, allant du programme d'enseignement imposé, fait le plus courant, à l'interdiction explicite d'enseigner certaines positions (passage en quelque sorte d'une censure négative et implicite à une censure positive et explicite), en passant par les censures doctrinales. Enfin, les personnes elles-mêmes peuvent être visées par des procès, des sanctions, des intimidations, ou subir ce que Bianchi appelle «la discipline des rapports interpersonnels ». Il évoque à cette occasion la subtilité des mécanismes de contraintes à l'intérieur du monde universitaire, qui pour être autonome n'en est pas moins régi par des lois propres, souvent tacites, qui conduisent les choix individuels et conditionnent la réflexion intellectuelle, de telle sorte que la liberté 
doctrinale ne peut se concevoir que dans des conditions sociologiques extrêmement spécifiques.

Le chapitre II pose le problème de l'efficacité de ces censures : Bianchi admet une large inefficacité, voire une contre-productivité de certains actes de censure, rejoignant en cela Libéra. Cependant il rappelle que les condamnations ont largement contribué, sinon à étouffer des doctrines, du moins à modifier considérablement leur réception, et la perception qu'on peut en avoir encore aujourd'hui, et que d'autre part, l'apparente inefficacité de la censure institutionnelle est aussi due à l'absence de prise en compte de toutes les formes de censure préalables, d'autocensure, de pressions diverses et même de simple restriction de l'espace du possible, ou plutôt du pensable, pour les philosophes médiévaux.

Enfin, le chapitre III ébauche une histoire de la thématisation de la liberté par ces philosophes. Répondant ici à Putallaz qui affirme que la liberté en tant que telle n'est ni vraiment discutée ou élaborée comme concept, ni a fortiori vraiment revendiquée par les médiévaux, Bianchi montre qu'il ne faut pas passer sous silence la construction progressive d'une libertas philosophandi, de Roger Bacon à Guillaume d'Ockham en passant par Arnaud de Villeneuve. La première partie aboutit à une vision moins iréniste que celle de l'historiographie traditionnelle des conflits intellectuels : la liberté de pensée des philosophes et la censure des autorités sont bien des réalités médiévales et non des concepts anachroniques pour Bianchi.

La deuxième partie développe l'exemple de la réception d'Aristote. Dans le premier chapitre, Bianchi prend une nouvelle fois comme point de départ la riche tradition historiographique (Mandonnet, Ehrle, Grabmann, Van Steenberghen). Cette dernière a voulu voir dans l'action de l'Église à l'égard d'Aristote une grande continuité, faite de prudence et de cohérence, visant à la domestication progressive de la pensée du Stagirite par les penseurs chrétiens. L'auteur veut prendre ses distances avec cette vision très téléologique, et rendre aux choix successifs de l'Église toute leur contingence et leur historicité : il parvient ainsi à une profonde et convaincante révision de l'interprétation traditionnelle. Les condamnations de 1210/1215 sont relativisées: Aristote est bien visé, mais l'intervention des censeurs ne se conclut pas par une interdiction de ses œuvres, mais seulement de certains livres, les « livres naturels ». Dès cette époque, l'Organon ou l'Éthique à Nicomaque sont partie intégrante de l'enseignement à l'Université. De même, en 1231, l'intervention du pape Grégoire IX doit aussi être ramenée à sa juste proportion. Certes, il confirme les décisions de 1210/1215, mais il n'est pas sûr qu'Aristote ait vraiment été la préoccupation du pape. Bianchi montre que l'interdiction partielle d'Aristote à la faculté des arts n'est en réalité qu'un élément secondaire dans un projet différent, celui de la réforme des études théologiques, qui ne devaient pas être envahies par la physique et la métaphysique. Grégoire IX ne se prononce pas sur la doctrine aristotélicienne elle-même, mais utilise une interdiction déjà existante pour limiter la propagation de domaines de réflexion qui ne sont pas du ressort de la théologie, et qui risquent de la détourner de son objet. Une nouvelle fois, la décision tient plus de la contingence historique que d'une attitude doctrinale cohérente de l'Église au sujet d'Aristote. 
Le chapitre II offre une révision tout aussi pertinente d'un autre conflit autour d'Aristote, qui aboutit à la condamnation des thèses ockhamistes à Paris entre 1339 et 1346 . Après avoir rappelé que le XIV siècle connut un renforcement de l'aristotélisme, Bianchi souligne la filiation entre la condamnation du statut de 1339 et le fameux statut de 1255 qui faisait d'Aristote le philosophe « officiel » de l'Université. Dans ce statut de 1339, Ockham n'est pas condamné doctrinalement : ce sont tous les cours, publics ou privés, à partir de ses œuvres qui sont proscrits. Le statut de 1340 n'aide pas à dissiper le flou, à tel point que la tradition historiographique a parfois hésité quant à savoir s'il était pro- ou anti-Ockham. Bianchi rappelle à juste titre qu'une fois encore, ce ne sont pas des doctrines qui sont rejetées dans ce texte, mais des pratiques d'enseignement et de commentaire. Ces conflits doivent être rapportés aux pratiques d'un corps enseignant, pour éviter de les déréaliser. Le texte de 1340 fixe les règles d'interprétation que doivent observer les universitaires, en particulier dans le commentaire d'Aristote - or ces règles vont contre les pratiques d'Ockham et sa lecture « hétérodoxe » du corpus aristotélicien. Bianchi peut ainsi conclure que ce ne sont pas la logique et l'épistémologie nominalistes qui sont attaquées, mais les pratiques herméneutiques d'Ockham, jugées trop radicales et dangereuses. Cette hostilité est confirmée par la lettre de Clément VI de 1346 adressé à l'Université de Paris, dans laquelle il défend l'aristotélisme contre Ockham pour ces mêmes raisons herméneutiques, achevant ainsi d'expulser Ockham de la filiation d'Aristote en faisant de son travail une alternative à combattre, et non une nouvelle interprétation du Philosophe. Au total, on voit les déplacements considérables que Bianchi fait subir à la tradition de la réception d'Aristote, apportant des solutions neuves à de nombreux dossiers restés problématiques, souvent à cause d'une approche trop abstraite et directement doctrinale des enjeux.

La troisième partie, «Autour de 1277 », se livre à un exercice du même type avec la fameuse condamnation de Tempier. Un premier chapitre rappelle l'antécédent du statut de 1272, pour montrer qu'il s'agit d'une première forme de solution au problème de la délimitation entre Faculté des arts et Faculté de théologie, mais que ce statut a été rédigé là aussi pour des raisons contingentes : la «scission des Normands » entraîne une fraction minoritaire des maîtres de la faculté des arts dans un conflit contre la majorité, qui prend alors ce statut restrictif sur la pratique de la philosophie pour donner des gages de son orthodoxie doctrinale contre les minoritaires, auxquels appartient Siger de Brabant, sans doute une des cibles du texte.

Bianchi montre ensuite qu'à la même question de la frontière entre artiens et théologiens, entre raisonnement naturel et Révélation, Boèce de Dacie et Siger de Brabant apportent également des réponses, qui ne sont pas celles du statut de 1272. Ce texte défendait à l'artien de s'occuper de toute question touchant la théologie, et lorsque la frontière était floue, trois attitudes étaient possibles : réfuter les arguments naturels s'ils allaient à l'encontre de la foi, déclarer simplement ces arguments faux et erronés, enfin ne pas parler de sujet posant ces difficultés - dans un ordre de renoncement croissant. Siger et Boèce proposent quant à eux deux solutions différentes, mais qui se rejoignent dans leur projet. Il s'agit non pas d'admettre une « double vérité », comme ils en ont été accusés, mais de relativiser 
les doctrines philosophiques par rapport à la foi. La foi est la vérité absolue, tandis que les raisonnements de la philosophie naturelle ne sont vrais qu'en fonction des prémisses posées, or ces prémisses ne sont jamais que celles d'un savoir d'ordre humain, qui ne peut donc pas prétendre atteindre la vérité absolue. La philosophie naturelle ne produit donc pas la vérité, mais des conclusions vraies dans son propre système de référence logique, qui est relatif.

On le sait, ces nuances n'ont pas été retenues par leurs adversaires, comme en témoigne la condamnation de 1277 examinée dans un ultime chapitre. Bianchi s'interroge une nouvelle fois sur la portée et les objectifs de cette condamnation dont on a parfois fait un tournant dans l'histoire de la pensée occidentale. En abandonnant une approche dogmatique pour se tourner vers la pratique, l'auteur constate que la condamnation a eu une postérité bien paradoxale : destinée à frapper les maîtres ès-arts, elle a surtout été retenue par les théologiens, qui s'y réfèrent souvent dans les décennies suivantes. Partant de ce constat, Bianchi démontre que cette condamnation est une étape d'un conflit plus large, qui n'oppose pas tant artiens et théologiens, que, au sein des théologiens, dominicains et franciscains. Ces derniers sont les vecteurs privilégiés de la propagation des articles condamnés dans l'Occident, ainsi que les inspirateurs de ce qui n'est qu'un des volets d'une attaque plus générale contre les Prêcheurs et surtout contre Thomas d'Aquin. Cette censure avait d'abord pour fonction de redéfinir les frontières de l'orthodoxie théologique d'une manière plus conforme aux positions franciscaines. La réutilisation des articles condamnés par Pic de La Mirandole, puis, en un sens contraire, par Campanella pour défendre Galilée montre finalement comment des idées, produites dans des circonstances spécifiques et selon des logiques pratiques autant que doctrinales, sont devenues des instruments intellectuels dont les usages successifs redéfinirent la signification.

Le livre de Bianchi n'est donc pas simplement un nouvel état de questions érudites sur les débats universitaires parisiens de la fin du Moyen Âge, c'est aussi un exemple méthodologique pour l'histoire intellectuelle. Le perpétuel souci de réintroduire l'historicité et la contingence lui permet de ne pas se prendre au piège de «monuments » comme la condamnation de 1277, sous lesquels il sait retrouver les documents, et la révision érudite de l'historiographie est au service d'une perspective qui réussit à montrer comment les logiques sociales et politiques et la construction du savoir intellectuel sont indissociables. 